\title{
Functional genomic characterization of immunogenic gluten proteins from oat cultivars that differ in toxicity for celiac disease
}

\author{
María de Lourdes Moreno Amador ${ }^{1}$, Julio Masaru Iehisa ${ }^{2}$, Carolina Sousa Martín ${ }^{1}$ and \\ Francisco Barro Losada ${ }^{3}$ \\ ${ }^{1}$ Departamento de Microbiología y Parasitología, Facultad de Farmacia, Universidad de Sevilla, Sevilla, Spain, \\ ${ }^{2}$ Departamento de Biotecnología, Facultad de Ciencias Químicas, Universidad Nacional de Asunción, San Lorenzo, \\ Paraguay and \\ ${ }^{3}$ Instituto de Agricultura Sostenible, CSIC, Córdoba, Spain
}

\begin{abstract}
Introduction: Oat human consumption has increased due to its nutritional value and its health benefits. Oat is a rich source of protein that contains high level of minerals, lipids, $\beta$-glucan, a mixed-linkage polysaccharide, which forms an important part of oat dietary fiber, and also contains various other phytoconstituents like flavonoids and sterols among others. Different pharmacological activities have been reported on oats like antioxidant, anti-inflammatory, antidiabetic or anticholesterolaemic.

The safety of oats in a gluten-free diet has been a topic of debate for several years. Previous studies suggested that oats may induce the immunological response in celiacs and others confirmed the impossibility of consuming oats habitually by its toxicity. Our research group found oat cultivars with different immunotoxic potential against G12 monoclonal antibody that may explain the different clinical responses observed in patients suffering from celiac disease. In this study we have characterized by massive sequencing the transcriptomes of non-toxic and toxic varieties.
\end{abstract}

Materials and Methods: The transcriptomes of both oat varieties were sequenced by Illumina HiSeq ${ }^{\mathrm{TM}} 2000$. To assemble the contents, criteria of overlap $>40 \%$ and similarity $>95 \%$ were used. The functional annotations were inferred by similarity to Uniprot reference proteins. The minimum similarity threshold required for annotating a transcript was a BLAST e value minor than $10^{-10}$. Uniref 90 was used for the selection of annotated proteins.

Results: We have found 17 and 11 locus in the non-toxic and toxic varieties, respectively. We selected a set of 239983 reference proteins downloaded from Uniprot belonging to the taxonomic nodes BEP clade. Only proteins representative of Uniref90 clusters were used. The identification of immunotoxic epitopes in the coding sequences were determined by alignment with the T-cell recognized canonicals, encompassing one to three mismatches. We identified a total of 24 epitopes with an average of 2 modifications in the genome of the toxic variety with respect to the non-toxic. The epitope variants DQ2.5-ave-1 $\beta$ and DQ2.5-glia- $\alpha 3$ were the most repeated.

Discussion: The presence of epitopes in the toxic oat variety that are not present in non-toxic variety could be related with the immunotoxic potential found in our previous assays and also with the different clinical responses in celiacs consuming oats. Preliminary results suggest that a depth study based on searching epitopes found in toxic oat variety could help to the identification of real oat varieties available for celiac patients, and therefore, their incorporation in improvement programs to obtain commercial lines without toxicity.

\section{Conflict of Interest}

There is no conflict of interest 